\title{
Investigation of the Algorithm to Find Defects in High-voltage Insulators for an Automated Thermal Imaging Control System
}

\author{
Alina Zaripova ${ }^{1}$, Damir Zaripov ${ }^{2,}$, and Alexander Usachev $^{2}$ \\ ${ }^{1}$ OOO «GDC Services», Kazan, Russia \\ ${ }^{2}$ Kazan State Power Engineering University, Kazan, Russia
}

\begin{abstract}
This paper describes a software algorithm for detecting defective insulating structures using infrared images. The defect detection criteria are based on a joint analysis of the mean and standard value of the brightness distribution of a set of insulators. The effectiveness of the developed criteria is substantiated by the results of laboratory tests of a significant number of insulators removed from high-voltage lines according to the results of thermal imaging diagnostics. Simultaneous analysis of thermograms of the same type of insulating structures according to the proposed algorithm is more effective in comparison with the subjective assessment of each of them separately, which was used earlier. In addition, this approach allows to reduce the time of analysis and decision-making based on the results of diagnostics.
\end{abstract}

\section{Introduction}

Infrared (IR) devices for detecting defects have become one of the main methods for inspecting electrical equipment. The growing needs for the reliability of electricity supply to the population increase the requirements for thermal imaging technology. At the present detection of faults largely depends on the experience of the personnel and has insufficient efficiency [1-12].

Most power equipment requires a large number of inspection personnel, many companies usually appoint external contractors to perform IR diagnostics. After these contractors have submitted their inspection reports with recommendations, the findings are evaluated against additional tests to confirm their validity and to approve repairs. The utility's own personnel therefore still need a lot of time to complete the complex evaluation work.

In recent years, research in this field has been focused on the automatic analysis of the thermal state of electrical equipment elements. The research is divided into various stages. The first step is to detect an element within the thermal image. At the second stage, statistical characteristics and other data on the thermal state of the corresponding element of electrical equipment are extracted. At the final stage, the calculated statistical characteristics are analyzed for the decision-making process [13]. This system uses a combination of artificial intelligence and digital image processing techniques. The system can receive any number of infrared images as input before performing automatic processing.

Defective elements are usually detects by determining which areas in infrared images have higher temperatures than these areas in good condition. The test results are classified into different categories based on the detected temperature levels, which indicates the level of severity of each situation [14].

Automatic diagnostics of infrared images using an intelligent system is still in its early stages. There are no problems when you need a bright object that is easily identifiable on a thermogram, for example, a contact connection of wires. It is much more difficult to automatically select an object and find a defect on it when its thermal contrast merges with the background and drowns into noise. This situation is typical when diagnosing insulating structures. They are characterized by temperature differences in tenths of a degree, due to low currents flowing in insulators.

If a weak localized hotspot on the insulator is not detected in time, the abnormal condition will continually deteriorate and eventually lead to breakdown. Conventional infrared targeting techniques are usually based on grayscale and texture differences [15-17]. However, when there is too much noise or weak targets in the image, these methods will not be able to accurately detect defects [18].

This paper describes and investigates in practice an algorithm for detecting defects on low-contrast thermal images of high-voltage insulation structures of overhead lines and substations.

\section{Algorithm research}

\subsection{Algorithm description}

The most difficult is automatically detect defects in suspended and supporting insulating structures installed on overhead lines and substations using a thermal image.

\footnotetext{
* Corresponding author: dzaripov@list.ru
} 
In the general case, the i-element of the insulating structure, for example an insulator in a string or a part of an extended insulator, can be represented as a parallel connection of the active resistance $\mathrm{R}_{\mathrm{i}}$ and the capacitance $\mathrm{C}_{\mathrm{i}}$. The expression for the active power $\mathrm{P}_{\mathrm{i}}$ released on the element when an alternating current I with a frequency $\omega$ flows through it has the following form $[19,20]$ :

$$
P_{i}=I^{2} \frac{R_{i}}{1+\omega^{2} C_{i}^{2} R_{i}^{2}}
$$

Formula (1) shows that $\mathrm{P}_{\mathrm{i}}$ has an ambiguous dependence on $\mathrm{R}_{\mathrm{i}}$. The active power has a maximum at $\mathrm{R}_{\mathrm{i}}$ equal to $1 / \omega \mathrm{C}_{\mathrm{i}}$. When $\mathrm{R}_{\mathrm{i}}$ is greater than $1 / \omega \mathrm{C}_{\mathrm{i}}, \mathrm{R}_{\mathrm{i}}$ increases with decreasing $\mathrm{Ri}$, and when $\mathrm{Ri}$ is less than $1 /$ $\omega \mathrm{C}_{\mathrm{i}}$, it decreases. For example, for a suspended insulation, the intrinsic capacitance of which lies in the range of $30-70 \mathrm{pF}$, the maximum heat release is achieved at $R_{i}$ values from 45 to $100 \mathrm{M} \Omega$, respectively. Since the active resistances of insulators can range from hundreds of $\mathrm{G} \Omega$ for serviceable ones to several $\mathrm{M} \Omega$ for "zero" ones, the same heat release power can characterize two significantly different insulation states serviceable and defective.

If we consider the insulating structure as a whole, then the serviceable and defective state of the element can be distinguished by the change in the total heat release of the entire insulating structure. If there is a decrease in the active resistance of the i-th structural element, then at the initial stage of degradation, when $\mathrm{R}_{\mathrm{i}}$ is significantly greater than $1 / \omega C_{i}$ and its insulation is still considered to be in good condition, this leads to an increase in heat generation only on it, since the stress distribution among the elements in the structure practically does not change. A further decrease in resistance and a transition to a defective state already leads to a redistribution of stress in the structure and an increase in heat generation on other éléments. Defect condition can be distinguished by changing in the spatial distribution of the intensity of thermal radiation throughout the structure. The values characterizing the distribution are the mean and standard deviation. Both of these distribution characteristics must be used to determine the condition of the insulation. A decrease in the active resistance of one of the elements of the insulating structure unambiguously leads to a certain increase in the average radiation intensity of the structure. However, its noticeable increase occurs with a decrease in active resistance on several structural elements. At the same time, the value of the root-meansquare deviation characterizing the spread of the intensities of the thermal radiation of the elements relative to the mean deviation can also be fixed at small values of the mean, when the radiation intensity changes only on one element, but with a decrease in the active resistance on several elements, a situation is possible when the standard deviation will be close to zero. The described principle was originally developed for the diagnosis of suspended and post porcelain insulators. However, it can be extrapolated to polymer insulators and surface contamination detection. During operation, polymer insulators are characterized by a breach of tightness and penetration of moisture under the shell closer to the end cap, which leads to a redistribution of voltage along the insulator and the appearance of local partial discharges with a corresponding increase in the mean and standard deviation of temperature values.

In dry conditions, contamination deposited on the surface of the insulator does not reduce its insulation resistance and does not affect the distribution of stress along the entire structure. However, when the deposited pollution is moistened (fog, dew, rain), the insulation resistance on the surface of the insulator will decrease, thereby changes the voltage distribution law on the insulators. At this point, contamination on the surface of the insulator will increase the leakage current and surface temperature of each insulator. The heating power $\mathrm{P}_{\mathrm{i}}$ and the increase in surface temperature $\Delta \mathrm{T}_{\mathrm{i}}$ of the $\mathrm{i}$-th element can be calculated, respectively, by formulas (2) and (3):

$$
\begin{gathered}
P_{i}=U_{i} I_{l c} \\
\Delta T_{i}=U_{i} I_{l c} / R_{t}
\end{gathered}
$$

In the formulas: $I_{l c}$ is the leakage current through the entire insulating structure; $\mathrm{R}_{\mathrm{t}}$ - thermal resistance, the same for all elements. Due to the uneven fouling of the insulators and their internal resistance, $\mathrm{U}_{\mathrm{i}}$ and $\Delta \mathrm{T}_{\mathrm{i}}$ will also differ along the insulating structure.

When implementing the algorithm in an automated system, the most difficult thing is to choose a threshold for the mean and standard deviation values of the brightness of insulators on thermograms. Two methods are possible. In the first case, an installation of a reference (serviceable) insulating structure can be identical to the one diagnosed and obtained in similar meteorological conditions. For reference isolation, the mean and standard deviation values will be the minimum. Another option is possible when diagnosing a large number of insulating structures under the same weather conditions. In this case, the threshold values can be set during subsequent computer analysis by taking the total mean and root mean square value for all thermograms. Determination of threshold values in this way is possible provided that the total number of defective insulators is significantly less than serviceable ones. This method of determining threshold values has found the greatest practical application. The above and the diagnostic technique were incorporated into the algorithms of the developed program.

\subsection{System}

The system contains a procedure for determining the mean and standard deviation in the spatial distribution of the intensity of self IR radiation of multi-element insulating structures. A thermogram is a picture where the brightness of each point is determined by its temperature. In turn, the temperature of one or other element in an insulating structure is uniquely related to the electrical power released on it. The algorithm for detecting defective structures is as follows: 
1. Highlighting on the thermogram of all insulators in a string or individual rod and support insulators;

2. Calculation of the mean and standard deviation in the distribution of the brightness of the points in the selected areas.

3. Determination of defective insulation by exceeding the obtained value of the mean or standard deviation of brightness over the set threshold values. The values of the average $\mathrm{P}_{\mathrm{av}}$ and standard deviation $\mathrm{D}$ in the distribution of the brightness of the insulators on the thermograms are calculated by the formulas:

$$
\begin{gathered}
P_{a v}=\sum_{i=1}^{n} P_{i} \frac{1}{n} \\
D=\left(\sum_{i=1}^{n} P_{i}^{2} \frac{1}{n}-P_{a v}^{2}\right)^{0.5}
\end{gathered}
$$

where $P_{i}$ - brightness i-th pixel, $n-$ the total number of all pixels on the insulation of the structure.

\subsection{Algorithm description}

The algorithm was tested using the thermograms of the garlands of porcelain insulators obtained during the diagnostics of the overhead lines of the railway. Since, according to the requirements of the developed method, when diagnosing a large number of insulating structures, it is necessary to observe the uniformity of meteorological conditions, the check was carried out on a sample of thermograms obtained during the examination at one site in one pass.

It is important to note that in order to obtain reliable results when working with a thermal imager, the following requirements must be observed.

1. The distance to the garland and the shooting angle are selected in such a way that the insulating structure in the image is in profile and all its elements are clearly visible.

2. When shooting, the thermal imager should be set to maximum sensitivity. The setting of the thermal imager for maximum sensitivity can be seen by the presence of characteristic hardware noises in the image of the insulating structure.

3. When shooting insulating structures in conditions where the temperature difference between the object and the background is large, for example, at night in clear weather against the background of the sky, it is necessary by adjustments to ensure that the brightness of all points in the image of the structure does not go beyond the dynamic range.

4. By adjusting the thermal imager, it is necessary to achieve the best visual quality of the image of the insulating structure. The thermal imager is adjusted once at the beginning of work and does not change subsequently, with the exception of adjusting the lens for focusing.

6. After entering the work area, it is necessary to measure the ambient temperature using a thermal imager or thermometer. Repeated temperature measurements should be carried out every hour of operation.

Before processing, all thermograms were put into separate folders on a computer in accordance with the areas where the work was performed. The data on the section of the overhead line "Shalega" - "Arya" are presented in table 1 as an example. The threshold values for the mean (113.5) and standard deviation (4.48) in the distribution of brightness in the thermal images of the garlands were set according to garland No. 8 in the table.

Separate strings of insulators, identified as defective and removed from the supports as a result of IR diagnostics, were checked in the laboratory with a megohmmeter and high voltage tests on a high-voltage installation. The results of laboratory testing of insulators are also reflected in Table 1. Of the thirteen strings of insulators listed in the table, two were not confirmed as defective during laboratory tests (No. 5,7). In these strings, all insulators withstood the $50 \mathrm{kV}$ AC test for one minute. A total of 105 insulator strings were tested. The presence of a defect was confirmed in 85 of them (81\%). This result is good, considering that when selecting defective insulating structures during surveys, there is a subjective factor of overestimating the number of defects. 
Table 1. Testing algorithm.

\begin{tabular}{|c|c|c|c|c|c|}
\hline № & Object & $\mathrm{P}_{\mathrm{av}}$ & $\mathrm{D}$ & Diagnosis & Tests \\
\hline 1 & & 138.35 & 12.46 & Defect & + \\
\hline 2 & & 130.32 & 4.68 & Defect & + \\
\hline 3 & & 129.69 & 9.14 & Defect & + \\
\hline 4 & & 152.84 & 7.08 & Defect & + \\
\hline 5 & & 158.54 & 4.47 & Defect & - \\
\hline 6 & & 161.33 & 6.01 & Defect & + \\
\hline 7 & & 162.36 & 6.26 & Defect & - \\
\hline 8 & & 113.15 & 4.48 & Normal & - \\
\hline 9 & & 141.13 & 3.51 & Defect & + \\
\hline 10 & A & 168.01 & 6.11 & Defect & + \\
\hline 11 & & 123.76 & 10.4 & Defect & + \\
\hline 12 & & 145.99 & 8.49 & Defect & + \\
\hline 13 & & 172.69 & 17.15 & Defect & + \\
\hline
\end{tabular}

\section{Conclusions}

Verification of the algorithm and the system shows the possibility of improving the diagnostic accuracy with joint computer processing of a set of thermograms of insulators structures in comparison with the subjective assessment of each of them separately, which was used earlier. In addition, this approach allows to reduce the time of analysis and decision-making based on the results of diagnostics. In addition to automating the processing of thermograms with insulators, the considered algorithm can be used to develop online systems for thermal imaging control of substation equipment, which are increasingly being used.

The work was supported by the Ministry of Science and Higher Education of the Russian Federation on fundamental scientific research «Distributed automated systems for monitoring and diagnostic the technical condition of overhead power lines and substations based on technology of broadband data transmission through power lines and the Industrial Internet of Things» (theme number 075-00063-20-02).

\section{References}

1. H. Yuan, X. Chen, Yu Wang, M. Su, State Detection of Electrical Equipment Based on Infrared Thermal Imaging Technology, Pattern Recognition and Computer Vision, Second Chinese Conference, PRCV 2019, Xi'an, China, Proceedings, Part I (8-11 November 2019)

2. J. He, T. Li, Infrared detection method and application of power equipment defect, Techn. Autom. Appl., 37 (10), 97-100 + 104 (2018)

3. X. Zhang, Z. Tang, X. Fei, et all, Power apparatus state detection and diagnosis based on infrared thermal imaging technology, J. State Grid Technol. College, 20 (05), 6-9 (2017)

4. H. Yuan, X. Chen, Y. Wang, M. Su, State Detection of Electrical Equipment Based on Infrared Thermal Imaging Technology, In: Z. Lin, et all (eds), Pattern Recognition and Computer Vision, PRCV 2019, Lecture Notes in Computer Science, Springer, Cham, 11857 (2019) DOI: 10.1007/978-3-030-31654-9_22

5. L. Kang, Substation equipment fault diagnosis based on infrared image processing, North China Electric Power University (2016)

6. Y. Wang, Y. Yin, J. Ren, Research on thermal state diagnosis of substation equipment based on infrared image, Advances in Mechanical Engineering (April 2019) DOI: 10.1177/1687814019828551

7. D.A. Yaroslavsky, D.A. Ivanov, V. Nguyen, V. Wire torsion measurement for the tasks of monitoring of the mechanical state of overhead power transmission line, Proceedings - 2020 International Conference on Industrial Engineering, Applications and Manufacturing, ICIEAM 20209111999 (2020)

8. R.G. Minullin, Y.V. Piskovatskiy, V.A. Kasimov, Model and Experimental Detection of Single Phase-to-Earth Faults of Overhead Conductors in 6-10 Kv Distribution Circuits by a Location Method, Proceedings - 2020 International Ural Conference on Electrical Power Engineering, UralCon 2020 9216267, p. 411-415 (2020)

9. S.R. Khasanov, E.I. Gracheva, M.I. Toshkhodzhaeva, S.T. Dadabaev, D.S. Mirkhalikova, Reliability modeling of high- 
voltage power lines in a sharply continental climate, E3S Web of Conferences 178,01051 (2020)

10. R.G. Minullin, V.A. Kasimov, P.V. Kononcev, Locational sensing equipment for detecting ice and damage on transmission lines, Proceedings 2020 International Conference on Industrial Engineering, Applications and Manufacturing, ICIEAM 20209112061 (2020)

11. D.A. Yaroslavsky, D.A. Ivanov, V. Nguyen, V. Wire torsion measurement for the tasks of monitoring of the mechanical state of overhead power transmission line, Proceedings - 2020 International Conference on Industrial Engineering, Applications and Manufacturing, ICIEAM 20209111999 (2020)

12. D. Ivanov, M. Sadykov, A. GolenishchevKutuzov, A., (...), T. Galieva, A. Arslanov, The application of the technology of sensor networks for the intellectualization of the overhead power transmission lines, E3S Web of Conferences 220,01071 (2020)

13. I. Ullah, R.U. Khan, F. Yang, L. Wuttisittikulkij, Deep Learning Image-Based Defect Detection in High Voltage Electrical Equipment, Energies, 13, 392 (2020)

14. Y. Chou, L. Yao, Automatic Diagnostic System of Electrical Equipment Using Infrared Thermography, 2009 International Conference of Soft Computing and Pattern Recognition, Malacca, 155-160 (2009) DOI: 10.1109/SoCPaR.2009.41

15. H.Q. Zhao, Infrared weak and small target extraction algorithm based on regional feature segmentation, Laser Infrared, 46, 308-312 (2016)

16. Z.H. Peng, S. Yang, Z.S. Wang, et all, Infrared target extraction based on mathematical morphology and automatic region growing, Infrared Technol., 36, 47-52 (2014)

17. X.G. Lu, An efficient infrared target extraction algorithm combined with radiation features, Laser Infrared, 46, 634-638 (2016)

18. Y. Wang, Y. Yin, J. Ren, Research on thermal state diagnosis of substation equipment based on infrared image, Advances in Mechanical Engineering (April 2019) DOI: $10.1177 / 1687814019828551$

19. A.D. Zaripova, D.K. Zaripov, A.E. Usachev, Criteria for equipment defects for thermal power substation control system, Power engineering: research, equipment, technology, 19 (5-6), 51-57 (2017) (in Russian) DOI: 10.30724/1998-99032017-19-5-6-51-57

20. Y. $\mathrm{Hu}, \mathrm{K}$. Liu, Inspection and monitoring technologies of transmission lines with remote sensing (2017) ISBN: 978-0-12-812644-8
21. R.M. Aleev, D.K. Zaripov, Method for remote diagnostics of multi-element insulating structure, RF Pat. 2305848, 10.09.2007 (2008). 